\title{
Asclepios: a Research Project-Team at INRIA for the Analysis and Simulation of Biomedical Images
}

\author{
N. Ayache, O. Clatz, H. Delingette, \\ G. Malandain, X. Pennec, and M. Sermesant \\ INRIA Sophia - Asclepios project-team, 2004 Route des Lucioles BP 93 \\ 06902 Sophia Antipolis Cedex, France \\ Nicholas.Ayache@sophia.inria.fr
}

\begin{abstract}
Asclepios ${ }^{1}$ is the name of a research project-team officially launched on November $1^{\text {st }}, 2005$ at INRIA Sophia-Antipolis, to study the Analysis and Simulation of Biological and Medical Images. This research project-team follows a previous one, called Epidaure, initially dedicated to Medical Imaging and Robotics research. These two project teams were strongly supported by Gilles Kahn, who used to have regular scientific interactions with their members. More generally, Gilles Kahn had a unique vision of the growing importance of the interaction of the Information Technologies and Sciences with the Biological and Medical world. He was one of the originators of the creation of a specific BIO theme among the main INRIA research directions, which now regroups 16 different research teams including Asclepios, whose research objectives are described and illustrated in this article.
\end{abstract}

\section{Introduction}

\subsection{The revolution of biomedical images and quantitative medicine}

There is an irreversible evolution of medical practice toward more quantitative and personalized decision processes for prevention, diagnosis and therapy. This evolution is supported by a continually increasing number of biomedical devices providing in vivo measurements of structures and processes inside the human body, at scales varying from the organ to the cellular and even molecular level. Among all these measurements, biomedical images of various forms increasingly play a central role.

Facing the need for more quantitative and personalized medicine based on larger and more complex sets of measurements, there is a crucial need for developing 1) advanced image analysis tools capable of extracting the pertinent information from biomedical images and signals; 2) advanced models of the human body to correctly interpret this information; and 3) large distributed databases to calibrate and validate these models.

\footnotetext{
${ }^{1}$ Asclepios was a Greek hero who later became the Greek god of medicine and healing. His most famous sanctuary was located in Epidaurus which is situated in the northeastern Peloponnese (from Ron Leadbetter).
} 


\subsection{Advanced Biomedical Image Analysis}

Tremendous progress has been made in the automated analysis of biomedical images during the past decades $[16,3]^{2}$. For instance, for rigid parts of the body such as the head, it is now possible to fuse, in a completely automated manner, images of the same patient taken from different imaging modalities (e.g. anatomical and functional), or to track the evolution of a pathology through the automated registration and comparison of a series of images taken at widely spaced time instants [20,42] (cf. Figure 1). It is also possible to obtain from a Magnetic Resonance image of the head an adequate segmentation into skull tissues, white matter, grey matter, and cerebro-spinal fluid [43], or to measure certain functional properties of the heart from dynamic sequences of Magnetic Resonance [2], Ultrasound or Nuclear Medicine images [22].
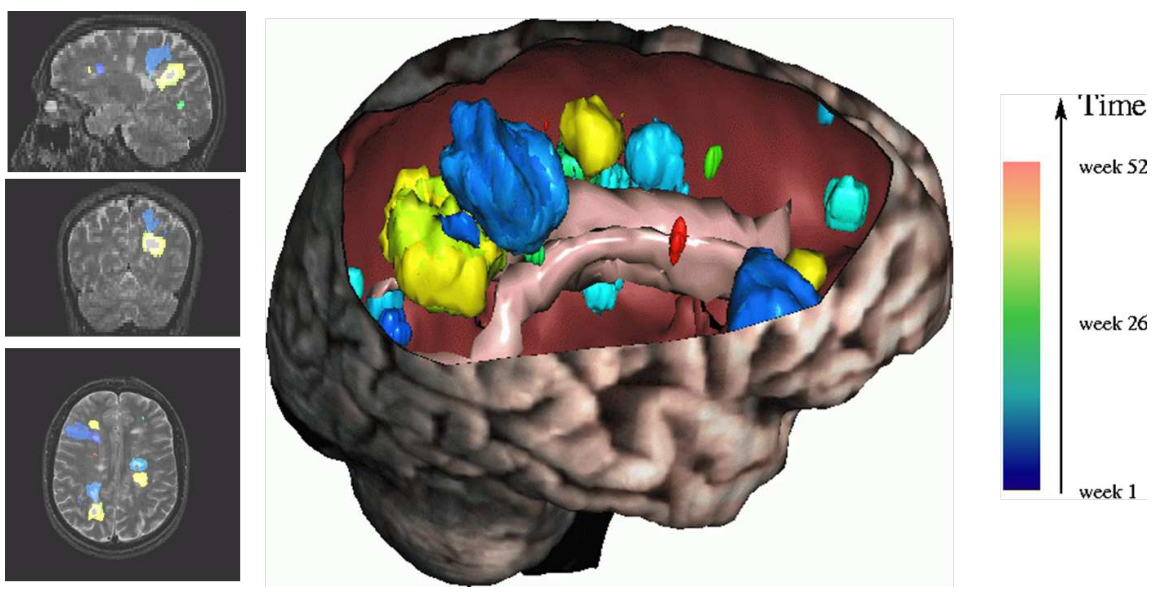

Fig. 1. Automatic detection of the evolution of multiple sclerosis lesions from a time sequence of 3-D magnetic resonance images of the head of a patient (from D. Rey et al. [34]).

Despite these advances one may note that statistical models of anatomy are still very crude, resulting in poor registration results in deformable regions of the body, or between different subjects. If some algorithms exploit the physical modeling of the image acquisition process, only a few actually model the physical or even physiological properties of the human body itself. Coupling biomedical image analysis with anatomical and physiological models of the human body

\footnotetext{
${ }^{2}$ One can gain a good understanding of the state of the art from the proceedings of the most recent conferences MICCAI'2006 (Medical Image Computing and Computer Assisted Intervention) or ISBI'2006 (Int. Symp. on Biomedical Imaging) as well as from the most recent issues of journals like IEEE Trans. on Medical Imaging or Medical Image Analysis (Elsevier).
} 
would not only provide a better comprehension of the observed images and signals, but would in addition provide efficient tools to detect anomalies, to predict the evolution of diseases and to simulate and assess the effect of therapies.

\subsection{Computational Models of Anatomy and Physiology}

Computational Models of the Human Body constitute an emerging and rapidly progressing area of research whose objective is to provide a better understanding of anatomical variability (Computational Anatomy) and of the major functions of the human body (Computational Physiology), as well as to provide effective algorithmic tools for their realistic numerical simulations $[4,14,5]$.

Quite advanced models have already been proposed to study at the molecular, cellular and organic level a number of physiological systems (e.g. cardiac, respiratory, digestive, nervous (central and peripheric), reproductive, etc. [24]). For instance, computational models of the heart have been proposed to interpret the cardiac electromechanical activity from medical images and electrophysiological measurements $[6,9]$, or to study the properties of physiological flows in blood vessels [33], in order to predict the appearance of cardiovascular diseases. Computational Models have also been proposed to explore the structures and the activity of the brain from anatomical and functional images and signals, for instance to better understand a number of brain diseases (e.g. Alzheimer's disease, Multiple Sclerosis, Creutzfeldt-Jakob disease, Epilepsy or Schizophrenia) [13]. Advanced models of abdominal organs including the liver [14] and the intestine [21] have been developed in the context of image-guided surgery (cf. Figure 2) and surgery simulation (cf. Figure 3). Other models have been developed to predict the evolution of cancerous lesions in various organs $[39,38$, $25]$.

It is necessary but not sufficient to develop, refine and validate such models. In addition, new methods must be designed to automatically fit the model parameters to a given person from the available biomedical signals (in particular medical images) and also from prior genetic information. Building such patientspecific models is a challenging goal which requires in particular the development of new data assimilation methods which can cope with massive numbers of measurements and unknowns.

\subsection{Large Distributed Databases}

Another important milestone towards progress in these directions is the development of large databases of subjects and patients, including biomedical signals and images as well as genetic information, and the development of specific tools to correlate, for instance, the shape and evolution of anatomical structures (phenotype) with the genetic information (genotype) and/or with a certain number of pathologies. The construction and exploitation of such databases require the development of specific measurement platforms which can regroup cutting edge 


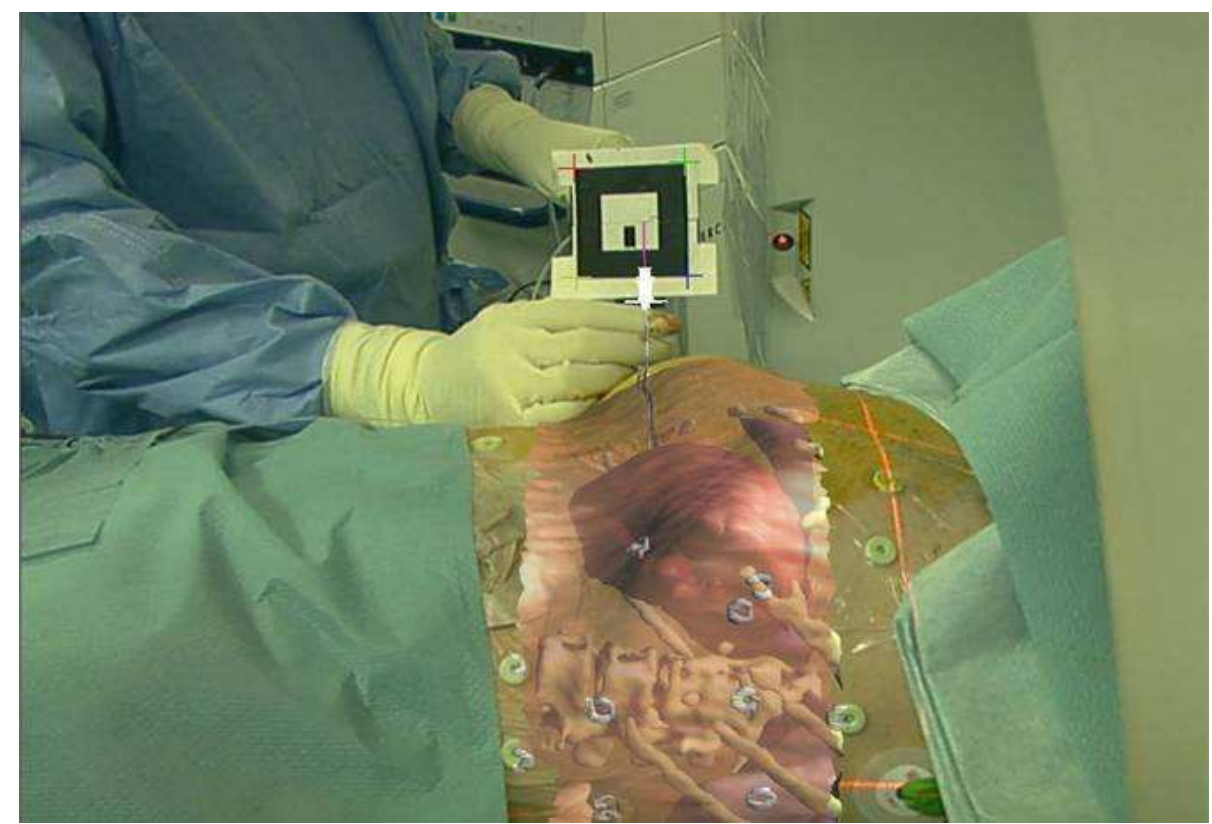

Fig. 2. Augmented reality for image-guided radio-frequency ablation of a liver tumor (from S. Nicolau, L. Soler et al. [28]).

imaging facilities with easy access provided to internal and external research teams (e.g. the Neurospin ${ }^{3}$ platform of CEA).

Huge computing power is already required to run advanced computational models in their direct mode (for prediction) or inverse mode (to adapt to a specific patient from biomedical measurements). The amount of required computing power to process large databases will require the development of grid-enabled algorithms capable of exploiting distributed computing power and data in large international networks [23].

\section{From Epidaure to Asclepios}

To address the above issues, the Asclepios research project-team was launched on November $1^{\text {st }}, 2005$ at INRIA Sophia Antipolis after final approval by the INRIA CEO Gilles Kahn. It built on the results of the previous research project-team Epidaure, initially launched at INRIA Rocquencourt in 1989 and later installed in Sophia-Antipolis in October 1992 thanks to the strong support of Gilles Kahn and Pierre Bernhard (at the time respectively VP for Science and Director of INRIA Sophia-Antipolis). The original scientific objectives were the quantitative

\footnotetext{
${ }^{3}$ URL of Neurospin: http://www.meteoreservice.com/neurospin/.
} 

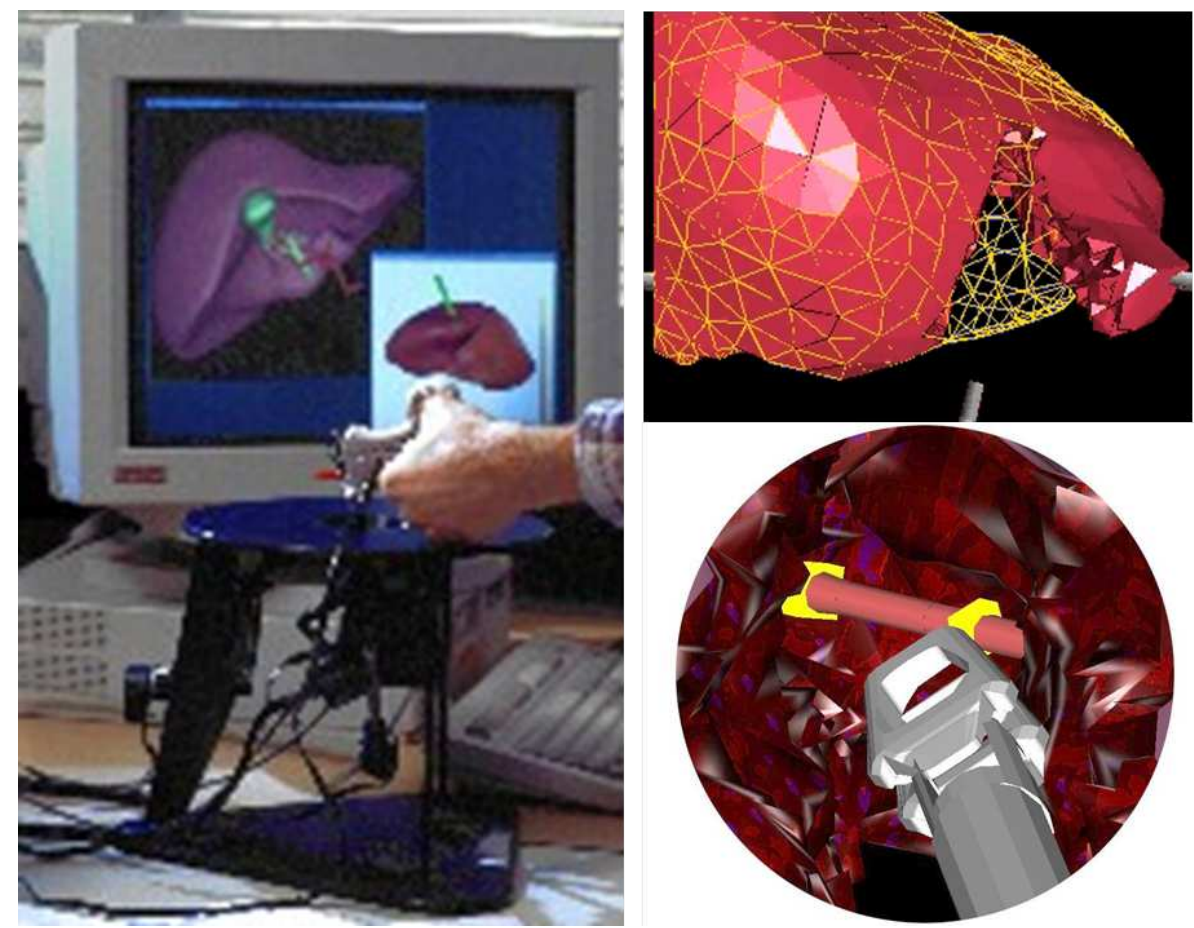

Fig. 3. Surgery simulation with visual and force feedback (cf. S. Cotin, G. Picinbono, C. Forest et al. $[12,32,18])$.

analysis of medical images and the coupling of medical imaging with medical robotics and surgery simulation.

Some of the major contributions of the Epidaure project were published in an invited article in the IEEE Transactions on Medical Imaging in November 2003 [3]. The Epidaure team has contributed, in association with a number of other teams, to the establishment of Medical Image Analysis and Simulation as a well distinct discipline in the field of computer science, with its own scientific community, its scientific peer-reviewed journals and conferences. In particular we contributed to the founding of the Medical Image Analysis journal (MedIA) and the MICCAI conference (Medical Image Computing and Computer Assisted Intervention), again with the encouragement of Gilles Kahn who attended the precursor conference CVRMed'95 (Computer Vision, Virtual Reality and Robotics in Medicine) organized by INRIA in Nice in April 1995, and the MICCAI'04 conference organized by INRIA in Saint-Malo in September 2004. 


\subsection{Personalized models for diagnosis and therapy}

If several of the problems listed in the original 1989 Epidaure research proposal have been solved, some important ones remain and new challenging problems have appeared. The research objectives of the Asclepios proposal take into account this situation and are organized around 5 research directions, namely: 1) Medical Image Analysis; 2) Biological Image Analysis; 3) Computational Anatomy; 4) Computational Physiology; and 5) Clinical and Biological Validation. Only directions 1 and (part of) 5 correspond to a continuation of the research objectives of the former Epidaure project, whereas directions 2, 3 and 4 correspond to novel objectives related to emerging problems.

Figure 4 attemps to summarize the overall objectives of the Asclepios project. The computational models of the human body that we consider often have four different primary components which we characterize as geometry, statistics, physics and physiology. In order to personalize a generic model, it is necessary to identify its parameters by confronting it to a set of patient-specific biomedical images and other biomedical signals. The personalized model can then, in turn, be projected back onto the images and signals to better interpret them, and to provide support for the diagnosis of disease. The personalized model can also be used to predict the evolution of disease, or to plan and simulate an intervention. This provides support for the prevention and therapy of diseases.

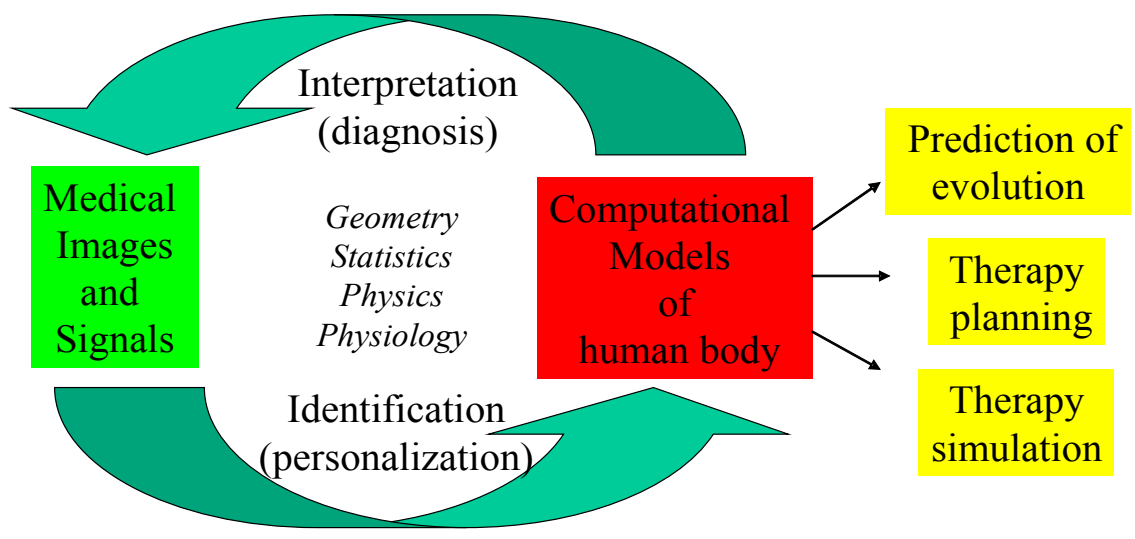

Fig. 4. Objectives of the Asclepios project-team.

In the remainder of this article we provide four illustrations of several aspects of current research in Asclepios respectively on: a) the automatic measurement of the variability of the geometry of some brain structures; b) the simulation of the growth of personalized brain tumors; c) the simulation of personalized electromechanical activity of cardiac ventricles; and d) the construction of large mosaics 
of microscopic molecular images acquired in vivo and in situ. We conclude with a last section.

\section{Measuring Brain Variability from Sulcal lines}

The objective of Computational Anatomy is the modeling and analysis of biological variability of the human anatomy ${ }^{4}$. Typical applications cover the simulation of average anatomies and normal variations, the discovery of structural differences between healthy and diseased populations, and the detection and classification of pathologies from structural anomalies.

Studying the variability of biological shapes is an old problem (cf. the remarkable book "On Growth and Form" by D'Arcy Thompson [40]). Significant efforts have been made since that time to develop a theory of statistical shape analysis, refer to [15] for a good synthesis, to a specific special issue of Neuroimage [41] or more recently to the first international workshop on the Mathematical Foundations of Computational Anatomy organized by Xavier Pennec and Sarang Joshi at MICCAI'2006 [30].

Computational Anatomy lies at the intersection of geometry, statistics and medical image analysis. A three-stage approach to computing statistics on anatomical shapes was proposed by M. Miller in [27]: 1) construction from medical images of anatomically representative manifolds based on feature points, curves, surfaces and volumes); 2) assignment of a point-to-point correspondence between these manifolds (possibly through a restricted class of geometric transformations, e.g. rigid, affine, diffeomorphism); and 3) generation of probability laws of anatomical variation from these correspondences.

We provide below an illustration of a similar approach applied to the study of the variability of certain brain cortical structures. Through a longstanding collaboration $^{5}$ with the LONI research group of UCLA (Pr. Paul Thompson), we developed a method to study the variability of the so-called sulcal lines, which are defined by neuroanatomists as the bottoms of brain cortical foldings. These lines can be extracted and labeled manually by experts (in our case on a database of 700 subjects), or automatically by expert systems such as the one developed at CEA by the group led by J.F. Mangin [35].

Figure 5 shows a typical set of sulcal lines extracted on the cortical surface of a brain of the LONI database. Together with V. Arsigny ${ }^{6}$ and P. Fillard, we developed an original approach to register all the images of the database to a common reference frame after an affine normalization, followed by an individual alignment of all homologous sulcal lines. This enabled us to compute for each sulcal line an average sulcal line, and along each of these lines a local measure of

\footnotetext{
${ }^{4}$ Cf. the summer school organized at UCLA in July 2004 by P. Thompson (UCLA) and M. Miller (Johns Hopkins) cf. www.ipam.ucla.edu/programs/mbi2004/.

${ }^{5}$ Cf. Associated Teams BRAIN-ATLAS http://wwwsop.inria.fr/epidaure/Collaborations/UCLA/.

${ }^{6}$ Vincent Arsigny received in 2007 the runner-up Gilles Kahn PhD prize for his research.
} 


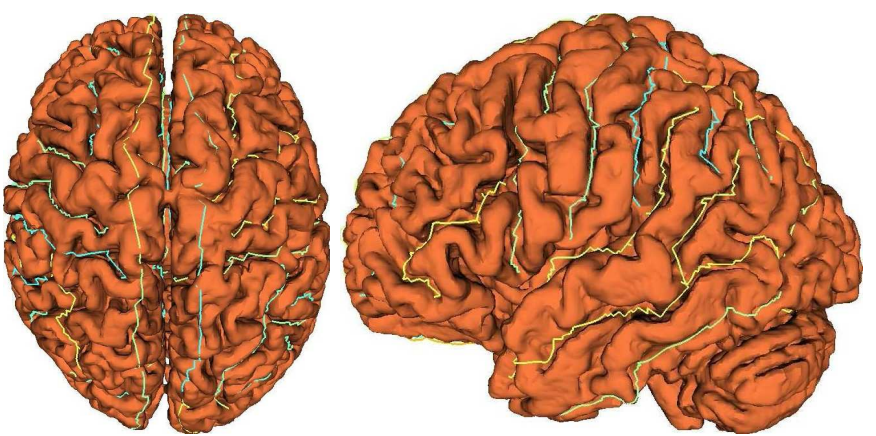

Fig. 5. Example of sulcal lines drawn on the cortical surface of one of the brains of the UCLA database (courtesy of P. Thompson).

variability under the form of covariance matrix computed at regularly sampled points.

As a result of new metrics defined in the space of Symmetric Positive Definite Matrices (also called "tensors" in our community), we were able to propose a method to extrapolate this local measure of variability to the whole cortex in a plausible manner $[29,1]$. We see in Figure 6 a color representation of the variability measured in various regions of the brain which quantitatively confirms previous observations made on a more qualitative basis by neuroanatomists. These results are published in [17].

Statistical information about variability can be used to help guide the superposition of a brain atlas to the brain images of a specific patient. This was shown with a different statistical database and in an image-guided radiotherapy context by $\mathrm{O}$. Commowick et al. in [11].

Another study, conducted during the PhD work of Jonathan Boisvert [8], concerned the statistical analysis of the variability of the scoliotic spine. By using an articulated model based on the relative position and orientation of successive vertebrae (through rigid body transformations), we were able to estimate the variability of the spine shape over a database of more than 300 patients. This was used to assess the evolution of the deformation during orthopedic treatments; moreover, the first four modes of variation appeared to be closely correlated to the usual clinical classification of scolioses, which reinforces the clinical interest of the chosen approach. Figure 7 illustrates a typical result.

\section{Simulation of Tumor Growth}

Combining anatomical, physical and physiological models of the human body is part of the Computational Physiology research field, to which the next two examples belong. The first concerns the simulation of diffusive tumor growth based on medical images. The objective is to identify the parameters of a generic 


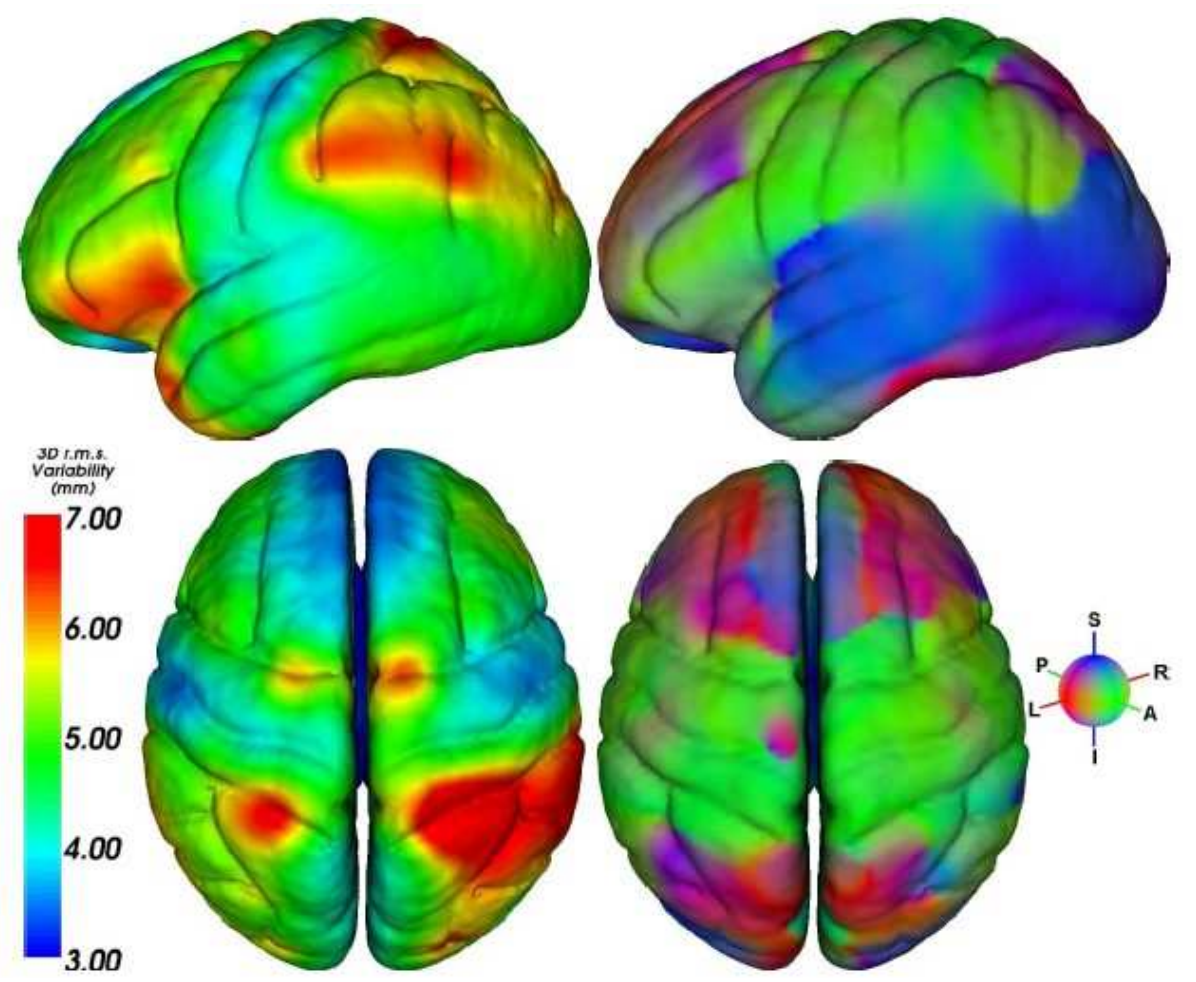

Fig. 6. Color maps showing the geometrical variability of the cortical structures extrapolated from the sulcal variability and a mathematical Riemannian framework. Left column shows the amplitude of the variability (hot colors mean high variations among subjects). Right column shows the main direction of variability. (color code: Red: leftright oriented tensor, Green: posterior-anterior oriented, Blue: inferior-superior oriented). Cf. P. Fillard, V. Arsigny et al. [17].

dynamic model from a sufficient number of observations on a specific patient, in order to better characterize the nature of the observed tumor. This imagebased characterization of the tumor aims at better predicting its plausible evolution, and anticipating the effects of possible therapies (e.g. radiotherapy, surgery, chemotherapy).

The work described in [10] includes three main levels of modeling: the first is geometrical and includes the extraction of the main structures of the head from a set of multisequence magnetic resonance images (MRIs). Among these structures are 1) the skull, 2) the gray and 3) the white matter of the brain, 4) the cerebrospinal fluid and 5) the falx cerebri. Figure 8 shows a typical representation of these structures. The accurate geometric description of these structures is important because the proliferation and migration of glial tumor cells strongly depend on the nature of the tissues and also on the orientation of the white matter fibers. 

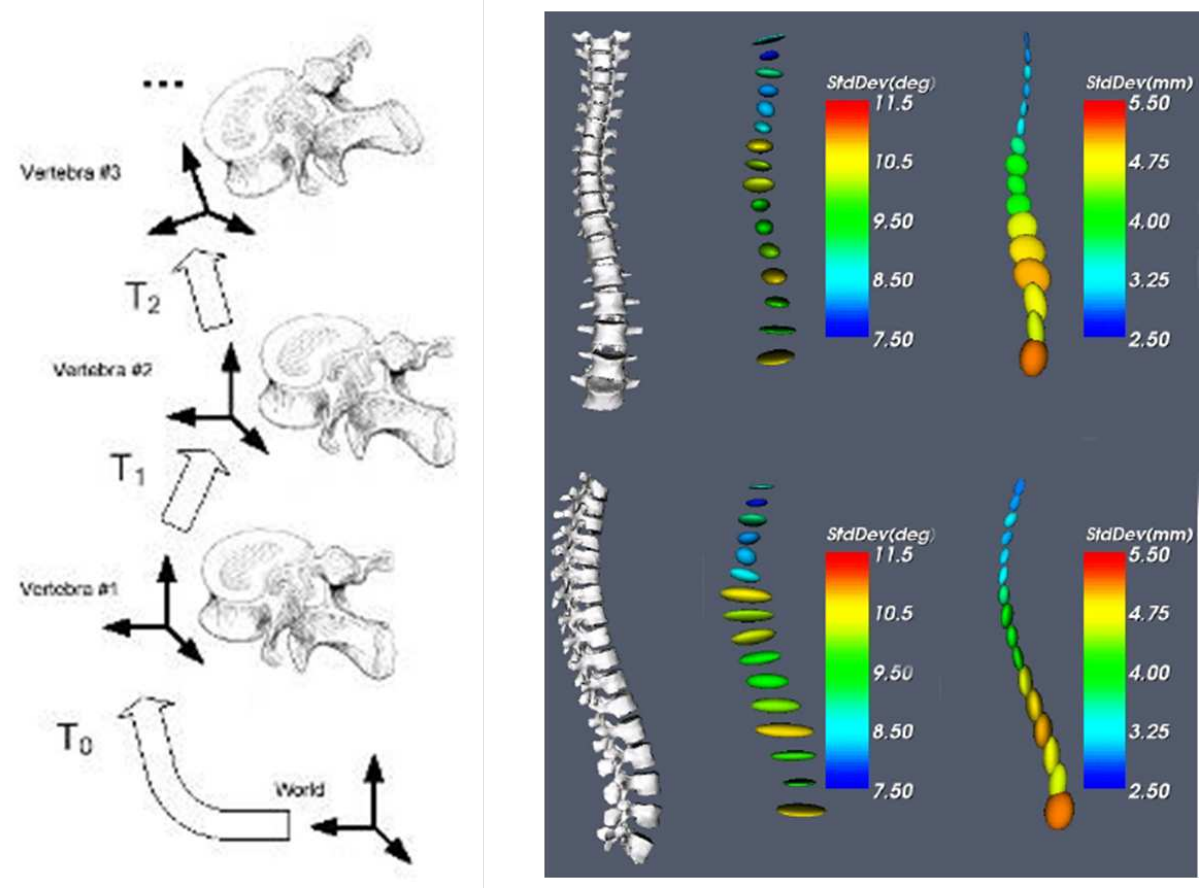

Fig. 7. Statistical spine model. From left to right: mean spine model reconstructed from a database of 300 patients with scoliosis, rotation and translation covariance measuring the local variability of each vertebra (color encodes the determinant of the covariance matrices). Top: Postero-anterior view. Bottom: lateral view. (cf. J. Boisvert [8])

The second level of modeling refers to biomechanics. The aim is to simulate the deformation of the brain induced by the tumor growth, also called the "mass effect". We built a finite element model of the previously extracted head structures under the hypothesis of inhomogeneous isotropic linear elastic behavior of the biological tissues. Linearity is a reasonable assumption to impose since the amplitude of the deformations is small. Inhomogeneity takes into account the mechanical variability of the tissues.

The third level of modeling concerns the physiopathology of the tumor itself. We consider a macroscopic description of the tumor cell density through a Fisher-Kolmogorov reaction diffusion equation. The reaction component is a second order polynomial which corresponds to a logistic proliferation law: exponential increase of number of cells followed by a reduced proliferation rate until an asymptotic value is reached. The diffusion component models the migration of tumor cells in their neighborhood. Because glioma cell migration is preferentially along the white matter fibers, the main bundles of white matter fibers are included in the model, through diffusion tensor MRI. The biomechanical coupling 
with the reaction-diffusion equation is introduced in the constitutive equation through a local pressure proportional to the tumor cell density.

Figure 9 shows a simulation of the progression of a glioblastoma during a six month period, with a good correspondence between observations and simulations. The identification of the parameters of the model followed an interactive and semi-automatic procedure. Currently it is the $\mathrm{PhD}$ work of E. Konukoglu [26] to identify automatically these parameters from the observations at two time points, and to measure the predictive power of the model against a third time point.

Once validated, we believe that such models could be used to better delineate for instance the dosimetry planning in radiotherapy, by proposing a margin outside of the visible contours of the tumor which would take into account the actual proliferation and diffusion parameters of the model, as well as the privileged directions of the main white matter fibers.

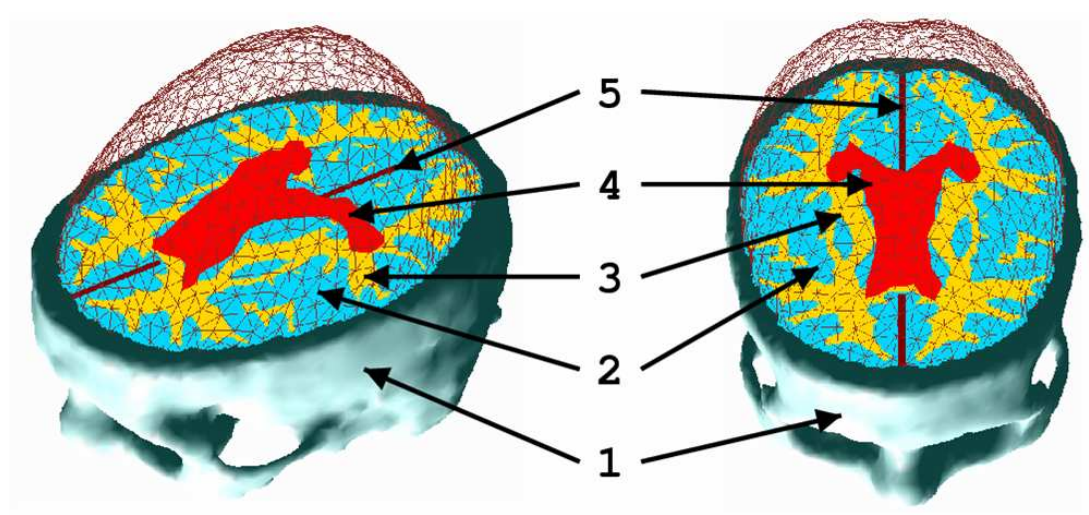

Fig. 8. The different tissues included in the biomechanical model of the head. 1 Skull. 2 Grey matter. 3 White matter. 4 Ventricles. 5 Falx cerebri.

\section{Personalized Electro-Mechanical Model of the Heart}

Building a personalized electro-mechanical model of the heart ventricles is an important precursor to the simulation and analysis of the electrical or mechanical activity of the heart of a specific patient.

We began research on this topic at INRIA about seven years ago through a collaborative action ICEMA ${ }^{7}$ funded by the scientific management of INRIA. This collaborative action was followed closely and supported by Gilles Kahn. It involved several project-teams at INRIA including Sosso, Caiman, Epidaure,

\footnotetext{
${ }^{7}$ www-rocq.inria.fr/sosso/icema2/icema2.html.
} 

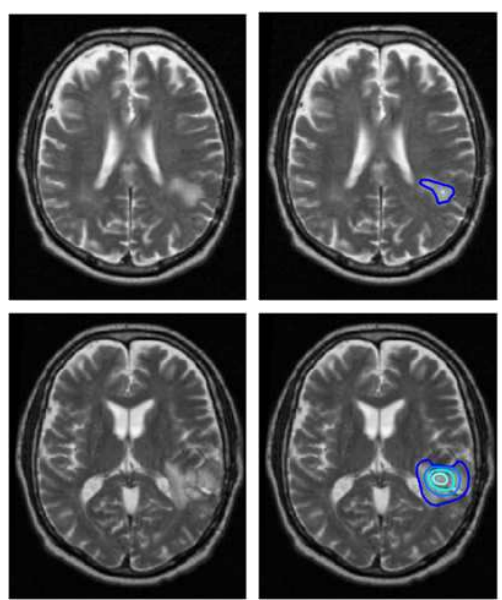

(A)

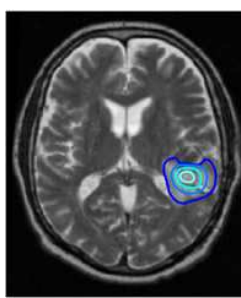

(B)
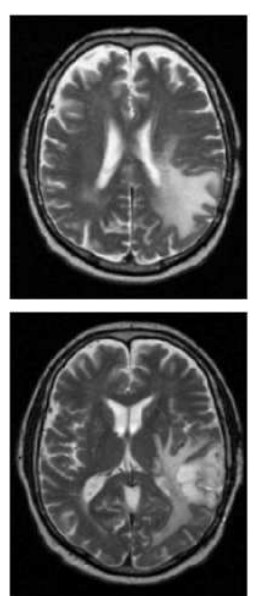

(C)
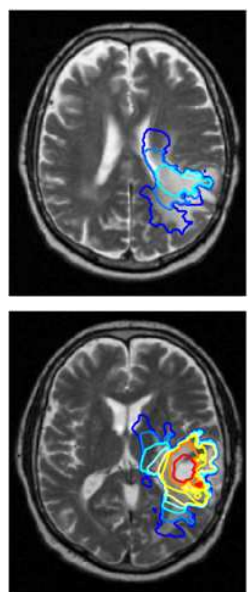

(D)

Fig. 9. Six months simulation of a glioblastoma growth. (A) T2 MRI of the patient, March 2002. (B) Previous MRI with superimposed tumor cell isodensity contours used for initialization. (C) T2 MRI of the same patient in September 2002 (6 months later). (D) Previous MRI with superimposed tumor cell isodensity contours simulated with the model.

Opale and Macs, as well as external research groups at NIH (E. Mc Veigh), Guy's Hospital (R. Razavi), and Philips (O. Gérard). This collaboration was reinforced more recently through a specific INRIA consortium called CardioSense $3 \mathrm{D}^{8}$, currently involving the INRIA project-teams Asclepios, Macs, Reo and Sisyphe, as well as the NIH, Guy's Hospital and Philips research groups.

The current models again include three levels of description [36]. The first is a geometrical description of the anatomy of the cardiac ventricles, which is adapted to the specific geometry of a given patient through image processing methods. The direction of the cardiac fibers is projected onto this geometric model by automatically mapping an average description which comes from a previous statistical analysis [31].

The second level of modeling is a macroscopic description of the evolution of the action potential of cardiac cells, which measures the difference between the extra-cellular and the intra-cellular electrical potentials. This evolution is modeled through a set of reaction diffusion equations (initially proposed by FitzHugh and Nagumo, and later refined by Aliev and Panfilov). These equations take into account the higher conductivity in the direction of the fibers at each point of the previous geometrical description, and enable the realistic simulation of the depolarization and repolarization waves within the cardiac tissues.

The third level of modeling is the electro-mechanical coupling which describes how the action potential variations actually control the contraction and the

\footnotetext{
${ }^{8}$ http://www-sop.inria.fr/CardioSense3D.
} 

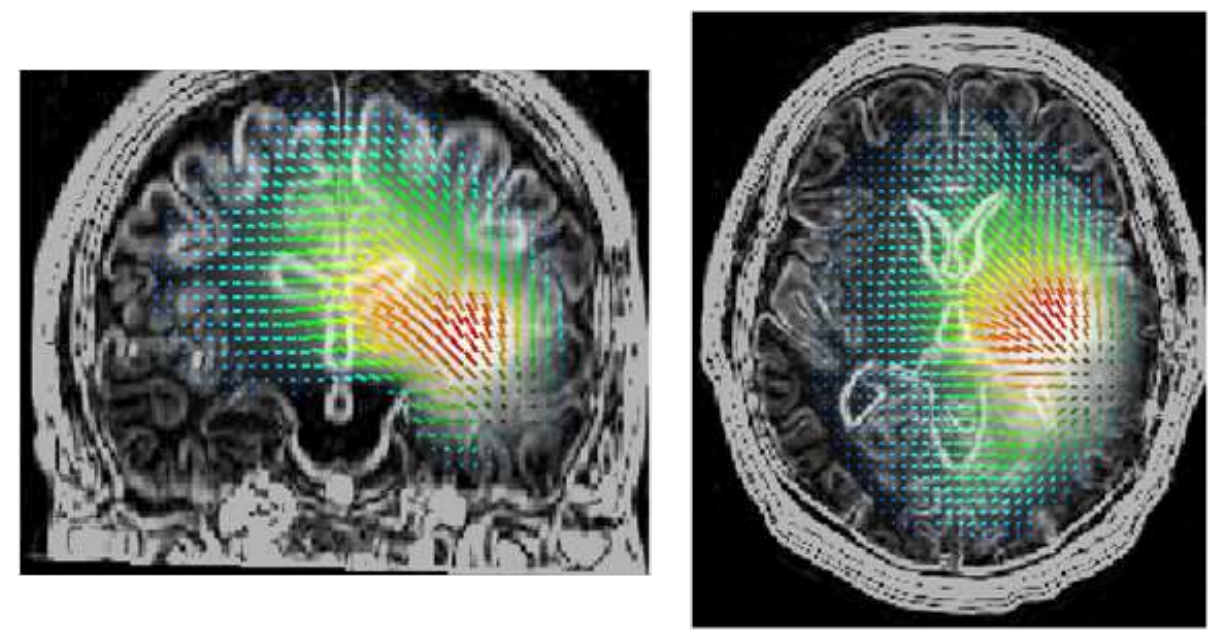

Fig. 10. The simulated displacement of the brain parenchyma due to the development of the previous tumor model in (left) coronal and (right) transverse views.

relaxation of the cardiac fibers. We chose a macroscopic model due to Bestel, Clément and Sorine [7]: a set of partial differential equations combines locally the behavior of an active elastic contractile element controlled by the previous action potential, with in addition two passive elastic elements respectively in parallel and in series. This model, initially inspired by the model of Hill-Maxwell is based on a multiscale analysis from the nanoscopic to the macroscopic scale. We see in Figure 11 an illustration of the realistic simulation of the contraction of a generic instance of this model.

The personalization of such a generic model must then be done through a set of specific measurements obtained through cardiac imaging (e.g. Ultrasound, Magnetic Resonance, Computed Tomography or Nuclear Medicine Imaging) as well as electrophysiology (typically the electrocardiogram or ECG). This is a difficult inverse problem which can be attacked through several strategies (cf. for instance [37]). Figure 12 shows the personalization of the model obtained through tagged MRI and specific electrophysiological measurements obtained with an endovascular procedure.

The future objectives of this research include the simulation of various therapies, like for instance Cardiac Resynchronization Therapy (CRT), or radiofrequency ablation procedures, or the effect of stem cells therapy.

\section{Building Large Mosaics of Microscopic Images}

The last example concerns microscopic imagery. During the past few years, we have initiated a research on the digital processing of microscopic images acquired in vivo and in situ. This was made possible through the development of new 


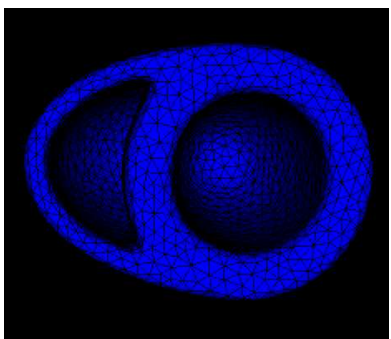

(a)

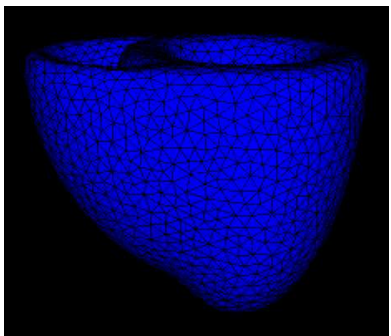

(d)

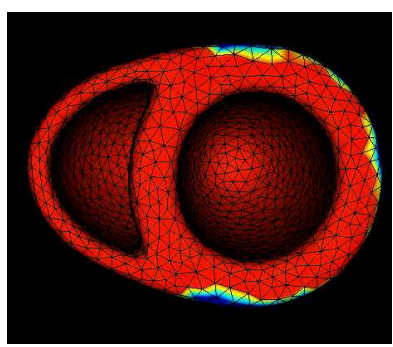

(b)

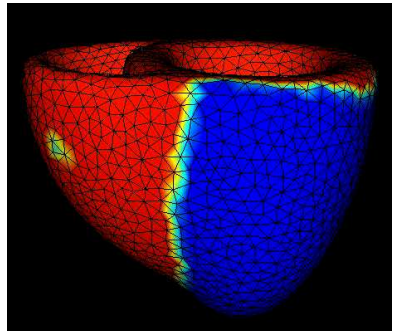

(e)

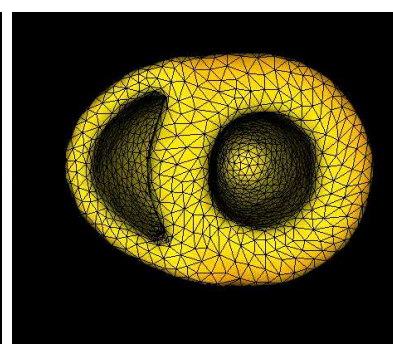

(c)

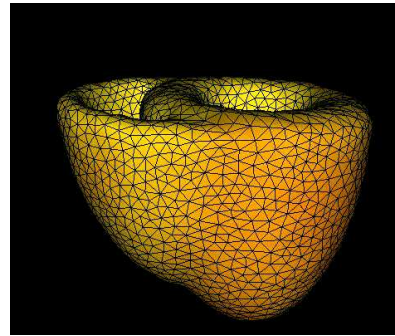

(f)

Fig. 11. Short axis (top row) and long axis (bottom row) views of an electromechanical heart model during end diastole (left column), ventricular depolarization (middle column) and end systole (right column).

imaging devices enabling the acquisition of confocal microscopic images at the end of a very thin optical probe. The probe can be introduced in the operator canal of an endoscope in order to provide images of the cellular architecture of suspicious tissues on line. This operation can also be seen as an "optical biopsy".

One of the issues with this type of imaging is the limited field of view of the microscopic images: because such images have a resolution of the order of a micron, the field of view is usually limited to a few hundreds of microns. This poses a problem when the analysis of the cellular architecture requires a larger field of view (this can be the case, for instance, when observing human colonic crypts), or when certain statistical measurements require a larger number of cells or vessels, or simply when it is necessary to reposition the probe exactly in a given location (when doing a temporal series of images at distant time points to study a slow dynamic phenomenon for instance). Through a collaboration with the Mauna Kea Technology company, T. Vercauteren [44] proposed during his $\mathrm{PhD}$ research a method to build a larger scene from the video sequence of microscopic images acquired during a smooth motion of the acquisition probe. The mosaic of images is obtained by replacing all of them in a common reference frame. This is a difficult problem because of the possible deformations induced by the motion of the probe and the non-rigid nature of the observed tissues. Figure 13 shows a typical example of the reconstruction of such a mosaic. Other 


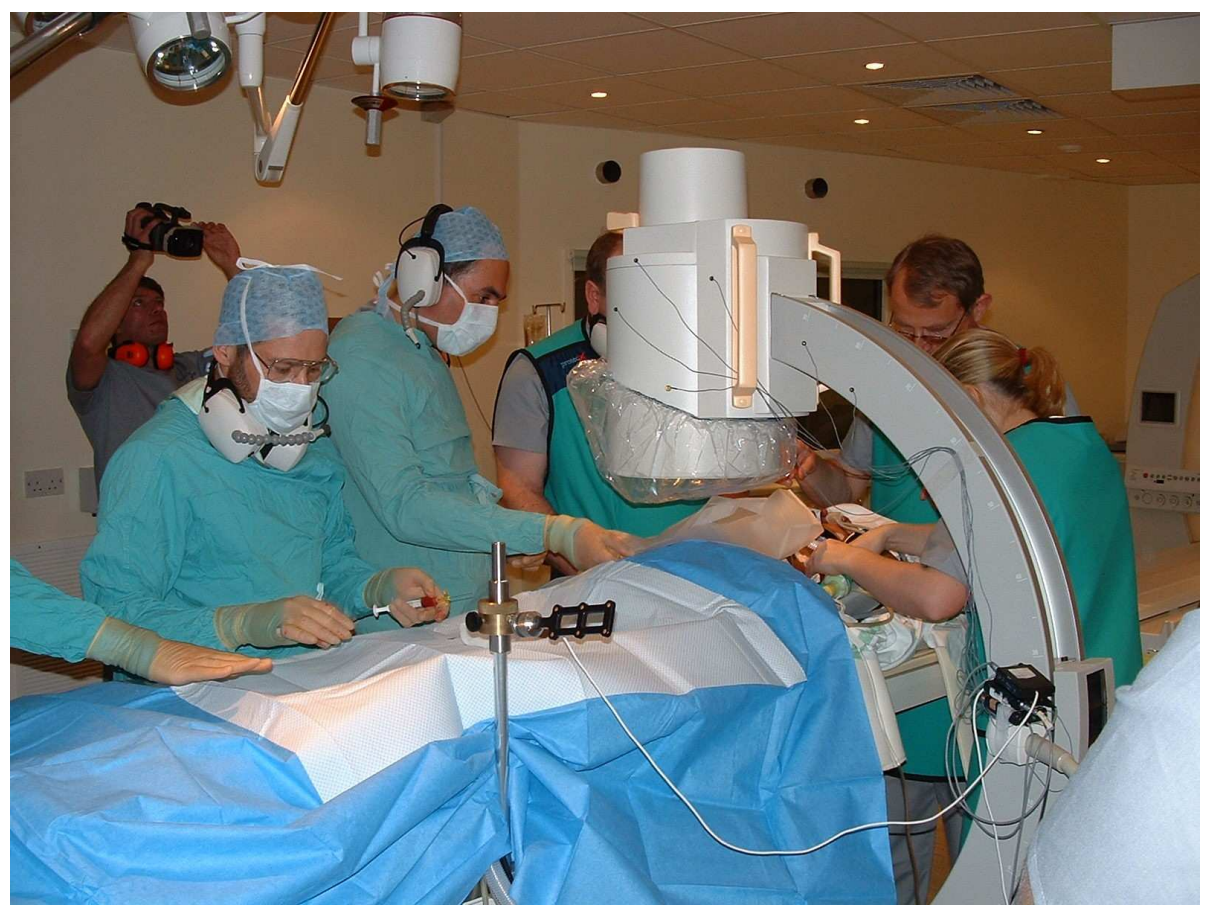

(a)
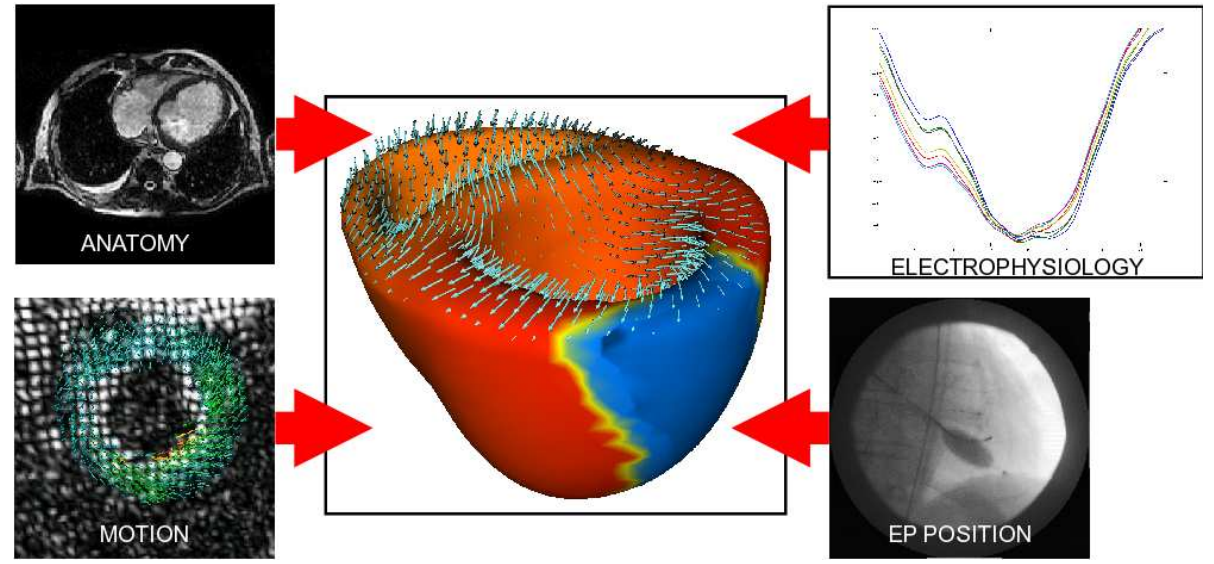

(b)

Fig. 12. (a) Intervention in the XMR room at Guy's Hospital (Pr. Reza Razavi) that allows to combine MRI and Xray acquisition. (b) Integration of XMR data providing anatomy, motion and electrophysiology measurements of a specific patient into the previous generic electromechanical model to get a personalized electro-mechanical cardiac model (cf. [37]). 
work on the construction of large mosaics of 3-D confocal images acquired from in vitro samples can be found in [19].

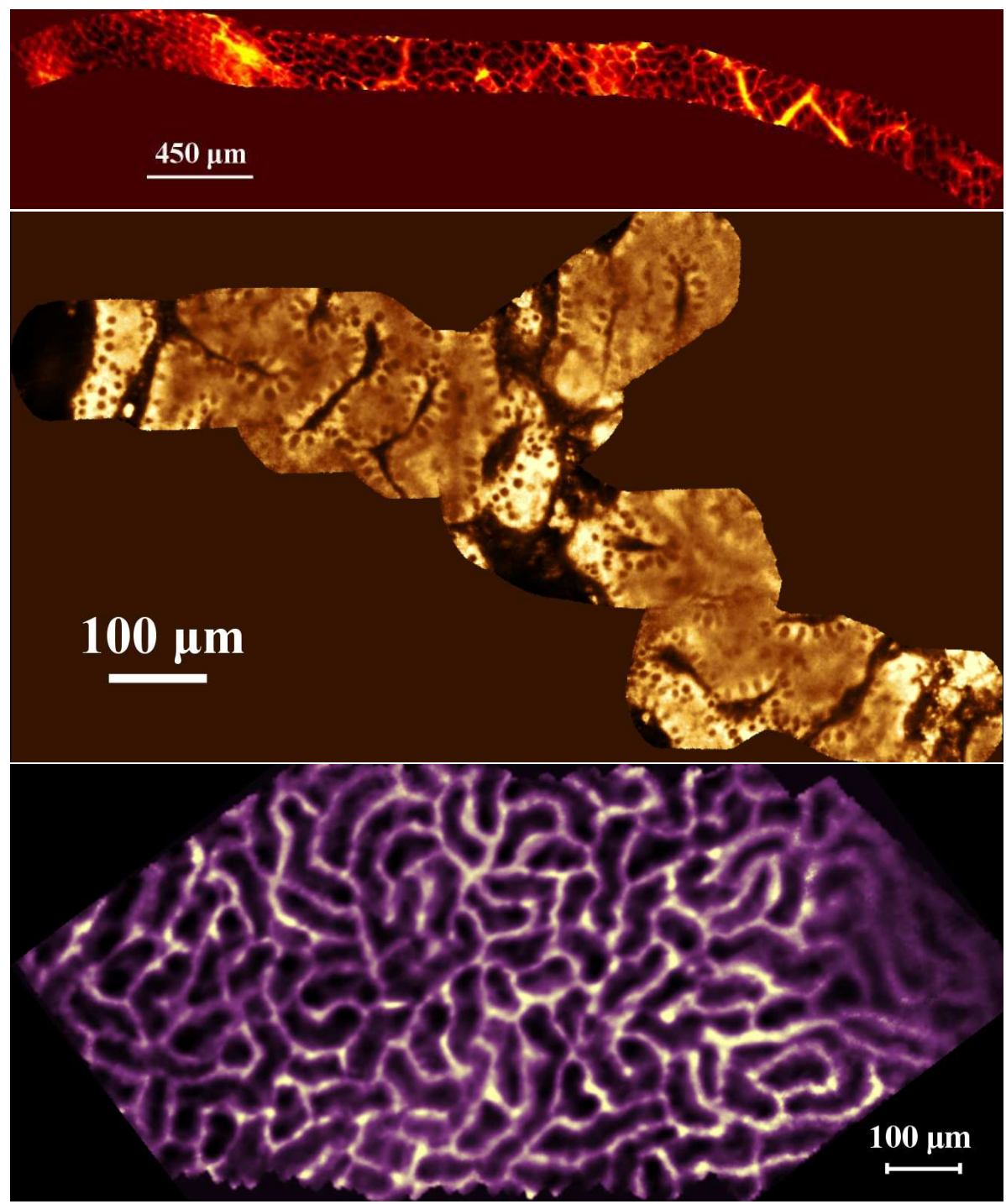

Fig. 13. Top : in vivo mouse colon vascularization after injection of FITC-Dextran high MW (300 input frames, Courtesy of M. Booth, MGH, Boston, MA); Middle : Ex vivo reflectance imaging of the human colon (mosaic includes 1500 input frames); Bottom : Microcirculation of the peritubular capillaries of a live mouse kidney with FITC-Dextran high MW (31 input frames). (cf. T. Vercauteren et al. [44]) 


\section{Conclusion}

We have outlined and illustrated in this article the current research objectives of the Asclepios project at INRIA, whose main application areas are in medicine and biology. The pharmaceutical domain is also an application area, as computational models of the human body can be exploited to better predict and quantify the effects of new drugs.

We believe strongly that the development of advanced biomedical image analysis methods combined with specific computational models of living systems will lead to a more profound understanding of the anatomy and physiology of the human body, and of the correlation between anatomical or physiological anomalies with the development of certain pathologies. We also believe that this research effort will be helpful to better exploit the huge amount of available biomedical signals (from in vivo molecular and cellular imaging to macroscopic organ imaging) as well as the genetic information potentially available on each patient.

An important clinical objective will be to increase significantly the potential for pre-symptomatic diagnosis and early treatment for a maximum medical efficiency. This research could also help the simulation and evaluation of various therapies, in particular traditional or minimally invasive surgery, radiotherapy, chimiotherapy, and also some currently experimental therapies like genetic or cellular therapy. It should also contribute to the promotion of image guided therapies.

Gilles Kahn strongly encouraged this research while he was the scientific director of INRIA Sophia-Antipolis, later the Vice-President for Science, and finally the CEO of INRIA. Through his numerous scientific interactions with the researchers involved, through his exceptional vision of the future, he stimulated this activity and gave those scientists the necessary confidence to explore new directions sometimes far away from their traditional background. For all these reasons we are proud to dedicate this article to the memory of Gilles Kahn.

\section{Acknowledgements}

The authors wish to thank all the past and current members of the Epidaure and Asclepios research project-teams, as well as their collaborators (both academic, clinical and industrial).

\section{References}

1. Vincent Arsigny, Pierre Fillard, Xavier Pennec, and Nicholas Ayache. Logeuclidean metrics for fast and simple calculus on diffusion tensors. Magnetic Resonance in Medicine, 56(2):411-421, August 2006. PMID: 16788917.

2. L. Axel, A. Montillo, and D. Kim. Tagged magnetic resonance imaging of the heart: a survey. Medical Image Analysis, 9(4):376-393, 2005.

3. N. Ayache. Epidaure: a research project in medical image analysis, simulation and robotics at inria. IEEE Trans. on Medical Imaging, 22(10):1185-1201, October 2003. 
4. N. Ayache, editor. Computational Models for the Human Body. Handbook of Numerical Analysis (Ph. Ciarlet series editor). Elsevier, 2004. 670 pages.

5. Nicholas Ayache, Jean-Pierre Boissel, Soren Brunak, Gordon Clapworthy, Guy Lonsdale, Jochen Fingberg, Alejandro Frangi, Gustavo Deco, Peter Hunter, Poul Nielsen, Matt Halstead, Rod Hose, Isabelle Magnin, Fernando Martin-Sanchez, Peter Sloot, Jaap Kaandorp, Alfons Hoekstra, Serge Van Sint Jan, and Marco Viceconti. Towards virtual physiological human: Multilevel modelling and simulation of the human anatomy and physiology. Virtual Physiological Human: White paper, EC - DG INFSO and DG JRC, 2006.

6. M.E. Belik, T.P. Usyk, and A.D. McCulloch. Computational methods for cardiac electrophysiology. In N. Ayache, editor, Computational Models for the Human Body, pages 129-187. Elsevier, 2004.

7. J. Bestel, F. Clément, and M. Sorine. A biomechanical model of muscle contraction. In W.J. Niessen and M.A. Viergever, editors, Proc. of International Conference on Medical Image Computing and Computer Assisted Intervention (MICCAI'01), volume 2208 of $L N C S$, pages 1159-1161. Springer, 2001.

8. Jonathan Boisvert, Xavier Pennec, Hubert Labelle, Farida Cheriet, and Nicholas Ayache. Principal spine shape deformation modes using Riemannian geometry and articulated models. In Proc of the IV Conference on Articulated Motion and Deformable Objects, Andratx, Mallorca, Spain, 11-14 July, volume 4069 of LNCS, pages 346-355. Springer, 2006. AMDO best paper award 2006.

9. D. Chapelle, F. Clément, F. Génot, P. Le Tallec, M. Sorine, and J. Urquiza. A Physiologically-Based Model for the Active Cardiac Muscle Contraction. In T. Katila, I.E. Magnin, P. Clarysse, J. Montagnat, and J. Nenonen, editors, Functional Imaging and Modeling of the Heart (FIMH'01), Helsinki, Finland, volume 2230 of $L N C S$. Springer, 2001.

10. Olivier Clatz, Maxime Sermesant, Pierre-Yves Bondiau, Hervé Delingette, Simon K. Warfield, Grégoire Malandain, and Nicholas Ayache. Realistic simulation of the $3 \mathrm{D}$ growth of brain tumors in mr images coupling diffusion with mass effect. IEEE Transactions on Medical Imaging, 24(10):1334-1346, October 2005.

11. Olivier Commowick, Radu Stefanescu, Pierre Fillard, Vincent Arsigny, Nicholas Ayache, Xavier Pennec, and Grégoire Malandain. Incorporating statistical measures of anatomical variability in atlas-to-subject registration for conformal brain radiotherapy. In J. Duncan and G. Gerig, editors, Proceedings of the 8th Int. Conf. on Medical Image Computing and Computer-Assisted Intervention - MICCAI 2005, Part II, volume 3750 of LNCS, pages 927-934, Palm Springs, CA, USA, October 26-29, 2005. Springer Verlag.

12. S. Cotin, H. Delingette, and N. Ayache. A hybrid elastic model allowing real-time cutting, deformations and force-feedback for surgery training and simulation. The Visual Computer, 16(8):437-452, 2000.

13. J. G. Csernansky, L. Wang, S. C. Joshi, J. T. Ratnanather, and M. I. Miller. Computational anatomy and neuropsychiatric disease: probabilistic assessment of variation and statistical inference of group difference, hemispheric asymmetry, and time-dependent change. NeuroImage, 23(Supplement 1):S56-S68, 2004. Special Issue: Mathematics in Brain Imaging.

14. Hervé Delingette, Xavier Pennec, Luc Soler, Jacques Marescaux, and Nicholas Ayache. Computational models for image guided, robot-assisted and simulated medical interventions. Proceedings of the IEEE, 94(9):1678-1688, September 2006.

15. I. L. Dryden and K. V. Mardia. Statistical Shape Analysis. John Wiley and Sons, 1998. 
16. J. Duncan and N. Ayache. Medical image analysis: Progress over two decades and the challenges ahead. IEEE Transactions on Pattern Analysis and Machine Intelligence, 22(1):85-106, 2000.

17. Pierre Fillard, Vincent Arsigny, Xavier Pennec, Kiralee M. Hayashi, Paul M. Thompson, and Nicholas Ayache. Measuring brain variability by extrapolating sparse tensor fields measured on sulcal lines. Neuroimage, 34(2):639-650, January 2007. Also as INRIA Research Report 5887, April 2006.

18. C. Forest, H. Delingette, and N. Ayache. Removing tetrahedra from manifold tetrahedralisation : application to real-time surgical simulation. Medical Image Analysis, 9(2):113-122, April 2005.

19. Céline Fouard, Grégoire Malandain, Steffen Prohaska, and Malte Westerhoff. Blockwise processing applied to brain microvascular network study. IEEE Transactions on Medical Imaging, 25(10):1319-1328, 2006.

20. Nick C Fox and J M Schott. Imaging cerebral atrophy: normal ageing to Alzheimer's disease. Lancet, 363(9406), 2004.

21. L. France, A. Angelidis, P. Meseure, M.P. Cani, J. Lenoir, F. Faure, and C. Chaillou. Implicit representations of the human intestines for surgery simulation. In Marc Thiriet, editor, Conference on Modelling and Simulation for Computer-aided Medicine and Surgery (MS4CMS'02), volume 12 of ESAIM: PROC, pages 1-7. European Series in Applied and Industrial Mathematics, 2002.

22. Alejandro F. Frangi, Wiro J. Niessen, and Max A. Viergever. Three-dimensional modeling for functional analysis of cardiac images: a review. IEEE Trans Med Imaging, 20(1):2-25, January 2001.

23. C. Germain, V. Breton, P. Clarysse, Y. Gaudeau, T. Glatard, E. Jeannot, Y. Legré, C. Loomis, I. Magnin, J. Montagnat, J.-M. Moureau, A. Osorio, X. Pennec, and R. Texier. Grid-enabling medical image analysis. Journal of Clinical Monitoring and Computing, 19(4-5):339-349, October 2005. PMID: 16328948.

24. P. Hunter. Computational physiology and the physiome project, 2004. "http://nbcr.sdsc.edu/mcmregistration/pdf/Peter_Hunter.pdf".

25. S Jbabdi, E Mandonnet, H Duffau, L Capelle, K R Swanson, M Pélégrini-Issac, $\mathrm{R}$ Guillevin, and H Benali. Simulation of anisotropic growth of low-grade gliomas using diffusion tensor imaging. Magn Reson Med, 54(3):616-624, Sep 2005.

26. Ender Konukoglu, Olivier Clatz, Pierre-Yves Bondiau, Hervé Delingette, and Nicholas Ayache. Extrapolating tumor invasion margins for physiologically determined radiotherapy regions. In Proc. of the 9th International Conference on Medical Image Computing and Computer Assisted Intervention (MICCAI'06), Part I, number 4190 in LNCS, pages 338-346, 2-4 October 2006.

27. M. I. Miller. Computational anatomy: shape, growth, and atrophy comparison via diffeomorphisms. NeuroImage, 23(Supplement 1):S19-S33, 2004. Special Issue: Mathematics in Brain Imaging.

28. Stéphane Nicolau, Alain Garcia, Xavier Pennec, Luc Soler, and Nicholas Ayache. An augmented reality system to guide radio-frequency tumor ablation. Computer Animation and Virtual World (previously the Journal of Visualization \& Computer Animation), 16(1):1-10, 2005.

29. Xavier Pennec, Pierre Fillard, and Nicholas Ayache. A Riemannian framework for tensor computing. International Journal of Computer Vision, 66(1):41-66, January 2006. A preliminary version appeared as INRIA Research Report 5255, July 2004.

30. Xavier Pennec and Sarang Joshi, editors. Proceedings of the First International Workshop on Mathematical Foundations of Computational Anatomy - Geometrical and Statistical Methods for Modelling Biological Shape Variability, Copenhagen, Denmark, October 2006. 
31. Jean-Marc Peyrat, Maxime Sermesant, Xavier Pennec, Hervé Delingette, ChenYang Xu, Eliot R. McVeigh, and Nicholas Ayache. A computational framework for the statistical analysis of cardiac diffusion tensors: Application to a small database of canine hearts. IEEE Transactions on Medical Imaging, 26(11):15001514, November 2007.

32. G. Picinbono, H. Delingette, and N. Ayache. Non-linear anisotropic elasticity for real-time surgery simulation. Graphical Models, 65(5):305-321, September 2003.

33. A. Quarteroni and L. Formaggia. Mathematical modeling and numerical simulation of the cardiovascular system. In N. Ayache, editor, Computational Models for the Human Body, pages 3-128. Elsevier, 2004.

34. David Rey, Gérard Subsol, Hervé Delingette, and Nicholas Ayache. Automatic detection and segmentation of evolving processes in 3D medical images: Application to multiple sclerosis. Medical Image Analysis, 6(2):163-179, June 2002.

35. Denis Rivière, Jean-Francois Mangin, Dimitri Papadopoulos-Orfanos, Jean-Marc Martinez, Vincent Frouin, and Jean Régis. Automatic recognition of cortical sulci of the human brain using a congregation of neural networks. Med Image Anal, 6(2):77-92, June 2002.

36. Maxime Sermesant, Hervé Delingette, and Nicholas Ayache. An electromechanical model of the heart for image analysis and simulation. IEEE Transactions in Medical Imaging, 25(5):612-625, 2006.

37. Maxime Sermesant, Philippe Moireau, Oscar Camara, Jacques Sainte-Marie, Rado Andriantsimiavona, Robert Cimrman, Derek L. Hill, Dominique Chapelle, and Reza Razavi. Cardiac function estimation from MRI using a heart model and data assimilation: Advances and difficulties. Medical Image Analysis, 10(4):642656, 2006.

38. R. Sierra, M. Bajka, and G. Székely. Pathology design for surgical training simulators. In International Symposium on Surgery Simulation and Soft Tissue Modeling, volume 2673 of LNCS, pages 375-384. Springer, june 2003.

39. K.R. Swanson, E.C. Alvord, and J.D. Murray. Virtual brain tumours (gliomas) enhance the reality of medical imaging and highlight inadequacies of current therapy. British Journal of Cancer, 86(1):14-18, 2002.

40. D'Arcy W. Thompson. On Growth and Form. Cambridge Univ. Pr., 1917.

41. P. M. Thompson, M. I. Miller, J. T. Ratnanather, R. A. Poldrack, and T. E. Nichols. Guest Editorial. NeuroImage, 23(Supplement 1):S1-S1, 2004. Special Issue: Mathematics in Brain Imaging.

42. PM Thompson, KM Hayashi, ER Sowell, N Gogtay, JN Giedd, JL Rapoport, GI de Zubicaray, AL Janke, SE Rose, J Semple, DM Doddrell, YL Wang, TGM van Erp, TD Cannon, and AW Toga. Mapping Cortical Change in Alzheimer's Disease, Brain Development, and Schizophrenia. NeuroImage, 23(supplement 1):S2-S18, 2004. Special Issue: Mathematics in Brain Imaging.

43. Koen Van Leemput, Frederik Maes, Dirk Vandermeulen, and Paul Suetens. A unifying framework for partial volume segmentation of brain MR images. IEEE Trans Med Imaging, 22(1):105-19, January 2003.

44. Tom Vercauteren, Aymeric Perchant, Grégoire Malandain, Xavier Pennec, and Nicholas Ayache. Robust mosaicing with correction of motion distortions and tissue deformation for in vivo fibered microscopy. Medical Image Analysis, 10(5):673-692, October 2006. Annual Medical Image Analysis (MedIA) Best Paper Award 2006. 